\title{
The embryonic stem cell microenvironment inhibits mouse glioma cell proliferation by regulating the PI3K/AKT pathway
}

\author{
Xiongjun $\mathrm{He}^{1}$, Jiahui Liu ${ }^{2}$, Xiaoran Wang ${ }^{3}$, Tengfei Liu ${ }^{4}$, Liu Yang ${ }^{3}$, Chaoyang $\mathrm{Li}^{3}$, Chenjie Wang ${ }^{5}$, \\ Ying Liu ${ }^{3}$, Xuan Sang ${ }^{3}$, Zhichong Wang ${ }^{3}$, Xiaohe $\mathrm{Lu}^{6} \wedge$
}

${ }^{1}$ Neurology, Shenzhen Hospital, Southern Medical University, Shenzhen, China; ${ }^{2}$ Department of Ophthalmology, Dongguan People's Hospital, Dongguan, China; ${ }^{3}$ Zhongshan Ophthalmology Center, Sun Yat-sen University, Zhongshan Ophthalmology Center, Sun Yat-sen University, Guangzhou, China; ${ }^{4}$ Institute of Gastrointestinal, The Sixth Affiliated Hospital (Gastrointestinal \& Anal Hospital), Sun Yat-sen University, Guangzhou, China; ${ }^{5}$ Joint Shantou International Eye Center, Shantou University and the Chinese University of Hong Kong, Shantou, China; ${ }^{6}$ Ophthalmology, Zhujiang Hospital, Southern Medical University, Guangzhou, China

Contributions: (I) Conception and design: Z Wang, X Lu, X He; (II) Administrative support: X Wang, J Liu; (III) Provision of study materials or patients: T Liu, L Yang; (IV) Collection and assembly of data: C Li, C Wang; (V) Data analysis and interpretation: Y Liu; (VI) Manuscript writing: All authors; (VII) Final approval of manuscript: All authors.

Correspondence to: Xiaohe Lu. Ophthalmology, Zhujiang Hospital, Southern Medical University, 253 Industrial Avenue, Haizhu District, Guangzhou 510282, China. Email: luxh63@163.com; Zhichong Wang. Zhongshan Ophthalmology Center, Sun Yat-sen University, laboratory 705, Building 3, Zhongshan Ophthalmology Center, Sun Yat-sen University, 54 Xianlie South Road, Guangzhou, China. Email: xxxxxxxxxxx.

Background: Glioblastoma multiforme (GBM) is the most common malignant tumor of the central nervous system, accounting for $48.6 \%$ of malignant tumors. The current standard treatment plan includes the widest range of safe surgical resection, supplemented by local brain radiotherapy and temozolomide concurrent chemotherapy; this can cause serious side effects. Even so, the median survival time of GBM patients is only 8 months, and the 5 -year survival rate is only $5.5 \%$. It is imminent to find new treatments. Early studies have shown that chicken and zebrafish embryos can reprogram cancer cells into a nontumorigenic phenotype through the embryonic microenvironment. However, the effect of embryonic stem cell microenvironment on GBM and its possible mechanism are not clear.

Methods: In this study, the glioblastoma cell line, U118, in the brain was investigated. There were four experimental groups: GB, GE, GA and GT. U118 cells were harvested after culturing for 72 hours. Cell proliferation, apoptosis, reactive oxygen species (ROS) were examined using vasculogenic mimicry assays, quantitative real-time polymerase chain reaction (QRT-PCR), western blotting (WB) and flow cytometry. The differences in the biological function of U118 cells and the PI3K/protein kinase B (AKT) signaling pathway were compared between the groups.

Results: Compared with the GB control group, the GE co-culture group and GT chemotherapy group showed reduced cell proliferation, increased apoptosis, increased ROS, as well as decreased or inhibited vasculogenic mimicry. Expressions of cyclin B1 and cyclin D1 were also notably reduced, while that of Bax, Bcl-2, p53, Caspase-3, GSK-3 $\beta$, p21, and p27 were significantly increased. Moreover, the expression of PI3K, AKT, and mTOR were markedly decreased, whereas expression of PTEN increased considerably. Also, the expression of positive regulatory factors significantly increased, however negative regulatory factors decreased in the GA group compared to the GE group.

Conclusions: The ESC microenvironment reverses glioma malignancy, partially via inhibition of the PI3K signaling pathway. Our study may have a significant impact and important clinical implications for cell therapy in the treatment of glioma.

Keywords: Embryonic stem cells (ESC); glioma; PI3K/AKT signaling pathway; microenvironment; cell therapy

\footnotetext{
^ ORCID: 0000-0003-2173-7290.
} 
Submitted Oct 28, 2020. Accepted for publication Jan 19, 2021.

doi: $10.21037 /$ tcr-20-3408

View this article at: http://dx.doi.org/10.21037/tcr-20-3408

\section{Introduction}

Glioblastoma multiforme (GBM) is the primary invasive malignant brain cancer, accounting for approximately $16 \%$ of all major cancers of the brain and spinal cord (1). Glioma has a poor prognosis due to the rapid growth and extensive infiltration of the tumor into nearby tissue. Currently, standard therapies include maximum surgical resection, postoperative adjuvant temozolomide (TMZ), and radiation therapy (2). However, since the boundary between GBM and normal brain tissue is unclear, it is not possible to completely remove the cancer cells. Despite improvements in surgical techniques, radical resection of the tumor mass is very difficult and incomplete, as infiltrated tumor cells inevitably remain in the surrounding tissue, resulting in a poor prognosis for GBM patients with a median survival of 15 months (1).

The mutation-centric model of cancer, which is essentially the idea that mutations cause cancer, has been challenged in recent studies. A representative "adaptive oncogenesis" model was recently introduced by Dr. DeGregori (3). This model views cancer from an evolutionary biological perspective, and has demonstrated that the microenvironment surrounding cancer cells is as significant in causing cancer development as mutations. A healthy microenvironment provides tumor-suppressive signals with controlled tissue homeostasis. However, once tissue homeostasis becomes uncontrolled, for instance by carcinogenic factors such as radiation, smoking, viral infection, and aging, the altered microenvironment can become a potent cancer promoter and favor the growth of cells with cancer-causing mutations (4). The microenvironment can also directly affect the phenotype of malignant cells without altering their genetic makeup. Therefore, in the treatment of cancer, it is not enough to consider only killing the cancer cells while ignoring the microenvironment that causes the cancerous changes. Given the pivotal role of the microenvironment in cancer initiation, progression, and metastasis, as well as the promising approaches for cancer prevention and treatment proposed by the evolutionary biological view, modulating the microenvironment may help to reverse a "cancer permissive" environment to a "cancer suppressive" one and maintain it.

Previous studies have suggested that the early embryonic microenvironment may reverse malignant melanoma cells into a non-tumorigenic phenotype (5). It has been demonstrated in chicken embryos (6), mouse blastocysts (7), and zebrafish embryos (5) that the embryonic microenvironment can reverse tumor cells to be non-tumorigenic. These findings indicate that modulating the microenvironment can reprogram cancer cells without damaging normal cells, thereby avoiding the side effects of standard anti-cancer therapies on normal organisms. However, with the development of the embryo, the ability to reverse the transformation of tumor cells into non-tumor phenotypes is reduced and is almost completely lost after birth (8). Attempts to reprogram tumor cells using adult stem cells have proved unsuccessful (9). The effect of reprogramming tumor cells using embryonic stem cell (ESC) conditioned medium (10) or ESC extracellular matrix (11) to mimic the embryonic microenvironment was much weaker than that observed in early embryos, presumably due to the lack of direct interaction between cancer cells and the ESC microenvironments These findings indicate that reprogramming tumor cells requires both the early embryonic microenvironment and cell-cell interactions. Therefore, ESC from blastocysts were selected for this study as they can provide a microenvironment similar to early embryos.

Researchers have successfully used ESC to simulate the microenvironment of embryos, confirmed their safety and efficacy in the treatment of leukemic mice (12), and found that the ESC microenvironment can reverse the degree of malignancy of uveal and cutaneous melanomas by downregulating the phosphoinositide 3-kinase (PI3K) pathway $(13,14)$. The present study aimed to investigate whether the ESC microenvironment can reverse the degree of malignancy of human glioma. In addition, the involved mechanism was studied to provide a new theoretical basis and treatment directions for the prevention and treatment of glioma. We present the following article in accordance with the MDAR reporting checklist (available at http:// dx.doi.org/10.21037/tcr-20-3408).

\section{Methods}

\section{ESC culture}

E14 mouse ESC were generously provided by Professor Peng Xiang of the Stem Cell and Tissue Engineering 
Research Center. ESCs were inoculated into a $25 \mathrm{~cm}$ culture flask at a density of $4 \times 10^{4} / \mathrm{cm}^{2}$, cultured at $37{ }^{\circ} \mathrm{C}$ and $5 \%$ carbon dioxide $\left(\mathrm{CO}_{2}\right)$, and passaged at the same density every 2-3 days.

\section{U118 cell culture}

U118 (ATCC ${ }^{\circledR}$ HTB-15TM) was purchased from American Tissue Culture Collection (ATCC, 10801 University Boulevard Manassas, VA 20110-2209 USA). U118 cells in good condition were plated in a Petri dish at a ratio of $1: 3$ (a density of approximately $3 \times 10^{4} / \mathrm{cm}^{2}$ ), cultured at $37^{\circ} \mathrm{C}$ and $5 \% \mathrm{CO}_{2}$, and passaged at the same density every $3-5$ days. There were four experimental groups:

(I) GB group (control group): U118 cells were cultured separately in GBM medium, and the U118 cells of interest were digested directly after 72 hours.

(II) GE co-culture group: ESC and U118 cells were mixed at a ratio of 1:3 in GBM medium and seeded in the same flask. After 72 hours of co-culture, the U118 cells of interest were isolated using a flow cytometry sorter.

(III) GA agonist co-culture group: ESC and U118 cells were mixed at a ratio of 1:3 in GBM medium and seeded in the same flask. After the cells were adherent for 2 hours, a PI3K pathway agonist [phosphatase and tensin homolog (PTEN) inhibitor], SF1670, was added $(2 \mu M)$. After 72 hours of co-culture, the U118 cells of interest were isolated using a flow cytometry sorter.

(IV) GT chemotherapy control group: After U118 cells became adherent in GBM medium, the chemotherapy agent temozolomide (TMZ) was added $(300 \mu \mathrm{M}$ TMZ was added 2 hours after the cells became adherent), and the U118 cells of interest were digested directly after 72 hours.

\section{3, 3'-dioctadecyloxacarbocyanine perchlorate (DIO) cell marker}

On a clean bench, a sterile pipette was used to remove the cell culture medium from the culture flask as much as possible. The cells were completely covered with the cell stain solution $\left(V_{y b r a n t}{ }^{\circledR}\right.$ DiD cell-labeling solution, Invitrogen.V22887), $5 \mu \mathrm{L}$ of DIO reagent per $1 \mathrm{~mL}$. After incubating for 20 minutes, the stain was discarded and washed three times with fresh U118 medium, and the cells were passaged according to conventional U118 cells.

\section{Flow cytometry sorting}

The cells were directly co-cultured in a culture flask, washed twice with phosphate-buffered saline (PBS), and mixed with TrypLE Express, Thermo 12604013 for 1-2 minutes. They were then centrifuged for 3 minutes at $1,500 \mathrm{rpm}$, the supernatant was discarded, and the cells were resuspended in the loading buffer. The cell concentration was adjusted to $1 \times 10^{7}$ cells $/ \mathrm{mL}$ and filtered through a $40 \mu \mathrm{m}$ cell sieve. DiO-labeled U118 cells selected by the flow cytometer were collected. The entire process was conducted away from light as much as possible.

\section{Flow cytometric determination of apoptosis}

After collecting $1 \times 10^{6} \mathrm{U} 118$ cells from each group, the cells were washed twice with PBS, centrifuged for 3 minutes at $1,000 \mathrm{rpm}$, and the supernatant was discarded. KGI AnnexinV-APC/7-AAD, YEASEN apoptosis detection kit reagents were added to the sample away from light and the samples were loaded into the flow cytometer.

\section{Cell proliferation experiments}

Each group of collected cells were centrifuged, resuspended, counted, and adjusted to a concentration of 5,000 cells $/ \mathrm{mL}$. $200 \mu \mathrm{L}$ of cell suspension was added to each well of a 96well plate, with five replicate wells per group. The plates were subsequently placed into an incubator. The culture medium was replaced with fresh cell culture medium containing Cell Counting Kit 8 (CCK8) $(10 \mu \mathrm{L}$ CCK8 per $100 \mu \mathrm{L}$ of fresh culture medium). The optical density (OD) was measured at $450 \mathrm{~nm}$ over 7 consecutive days using a multifunction microplate reader. The experiment was repeated in triplicate.

\section{Reactive oxygen species (ROS) measurement}

2, 7-Dichlorodihydrofluorescein diacetate (DCFH-DA) was diluted to a final concentration of $10 \mu \mathrm{mol} / \mathrm{L}$ in serumfree medium. The cells were collected at a concentration of $1 \times 10^{6}$ cells $/ \mathrm{mL}$ and resuspended in pre-diluted DCFH-DA. They were then incubated at $37^{\circ} \mathrm{C}$ for 20 minutes, and then washed three times with serum-free cell culture medium to completely remove the DCFH-DA that did not enter the cells. Each group of GBM cells was examined by flow cytometry within 30 minutes. 
Table 1 Primer sequences

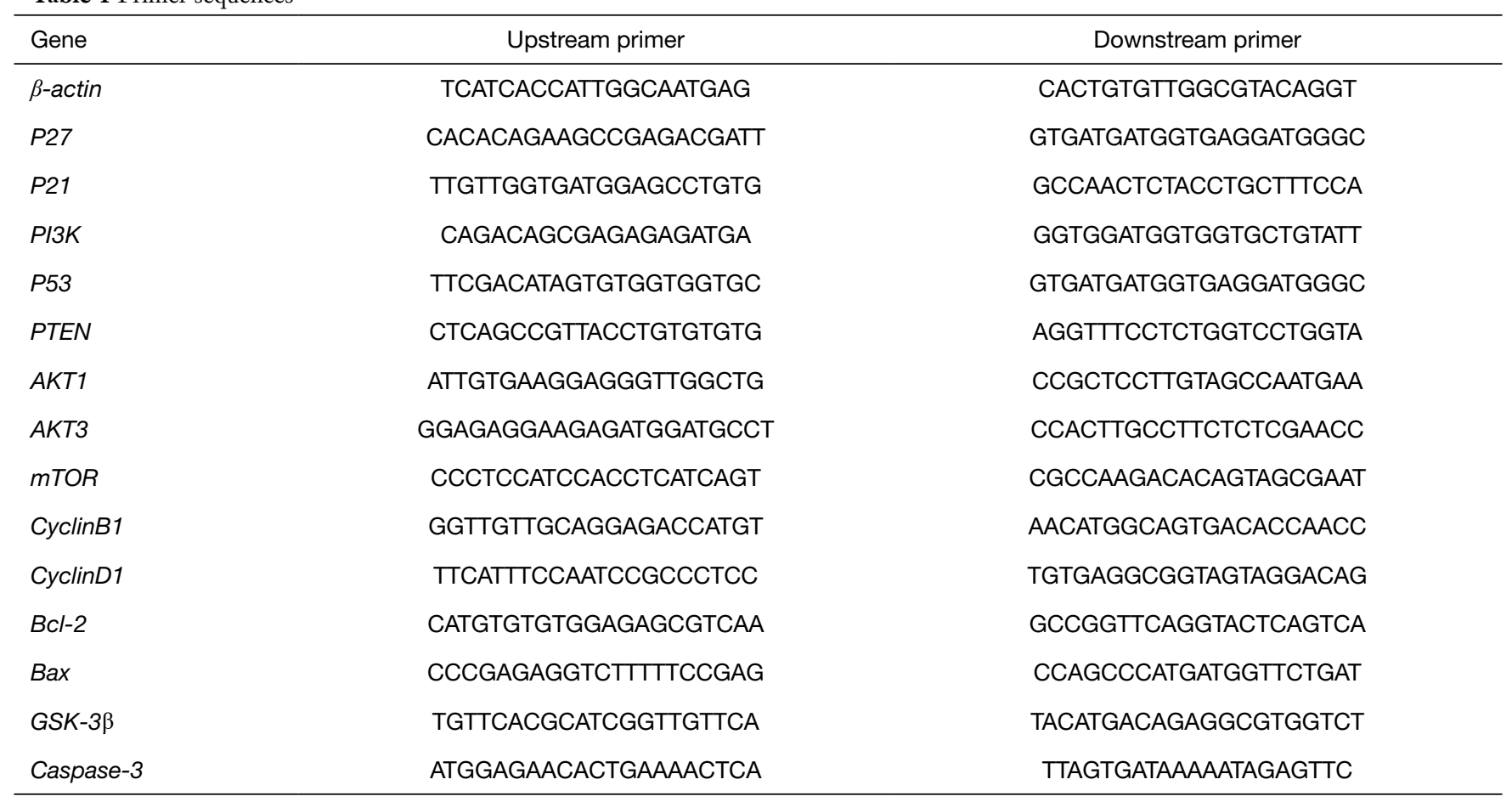

\section{Angiogenesis mimetic experiment}

After pre-cooling, the 96-well plate was pipette, tipped at $-20^{\circ} \mathrm{C}$, melted on Matrigel ice, placed on ice, and $30 \mu \mathrm{L}$ Matrigel was added to each well After 10 minutes in the refrigerator, it was then placed in the incubator for 30 minutes. Subsequently, the U118 cells were collected, the cell density was adjusted to $8 \times 10^{5}$ cells $/ \mathrm{mL}$, and $200 \mu \mathrm{L}$ of the cell suspension was taken into the spare wells coated with Matrigel, each group of three complexes. After 12 hours, the formation of angiogenic mimicry was observed with a light microscope, and the number of tubular structures in the same size of five medium-magnification fields was photographed and recorded. This experiment was repeated three times.

\section{Ribonucleic acid (RNA) extraction and semi-quantitative real-time polymerase chain reaction (QRT-PCR) analysis}

The total RNA of the cells was extracted using TRIzol reagent (Ambion), and the housekeeping gene, ACTIN, was used as an internal reference. First, using a complementary deoxyribonucleic acid (cDNA) synthesis kit in a $20 \mu \mathrm{L}$ reaction system, single-stranded cDNA was synthesized from $1 \mathrm{mg}$ of total RNA. The messenger RNA (mRNA) expression of $\triangle \mathrm{Np} 63, \mathrm{ABCG} 2$, integrin $\beta 1, \mathrm{CK} 3$, and other genes were detected by semi-quantitative polymerase chain reaction (PCR). Primers were designed using Primer 5 software (see Table 1 for details).

\section{Western blotting}

After treatment of each group of cells for 3 days, the collected cells were tested for expression of PI3K, AKT, mTOR, Bcl-2, Bax, etc., and were operated according to the previously described method. The antibodies used were as follows: anti-PTEN (ab32199, Abcam), anti-p53 (2542 CST), anti-PI3K (ab186612, Abcam), anti-p-PI3K (ab182651,Abcam), anti-AKT1/2/3 (ab179463, Abcam), anti-p-AKT(s473) (ab81283, Abcam), anti-mTOR (2983, CST), anti-p-mTOR(s2448) (5536,CST), anti-cyclin B1 (ab181593, Abcam), anti-Bcl-2 (ab32124, Abcam), anticyclin D1 (ab134175, Abcam), anti-Bax (ab77566, Abcam), anti-p21 (ab109520, Abcam), anti-Caspase-3 (ab32351, Abcam), anti- GSK-3 $\beta$ (ab75814, Abcam), anti- $\beta$-actin (ab8226, Abcam), and anti-p27 (ab32034, Abcam), antiJNK1 (ab199380, Abcam), anti-ASK1 (ab45178, Abcam), anti-p38 (ab182453, Abcam), anti- phosphol-p38 (ab178867, Abcam). The $\beta$-actin was used as an internal reference for 
this study.

\section{Statistical analysis}

All data in this study were analyzed using SPSS 11.0 software. All data were expressed as mean \pm standard deviation. Group data were statistically analyzed using the analysis of variance (ANOVA) method, and $\mathrm{P}<0.05$ was considered statistically significant.

\section{Results}

\section{ES microenvironment inhibited the growth of U118 cells}

As observed using an inverted microscope, ESC cultured alone grew as round or elliptical colonies with smooth and clear margins. The cells in the clonal colonies were small with large nuclei and little cytoplasm, and with a tight arrangement and unclear boundaries between cells. U118 cells cultured alone had an irregular long fusiform shape and were translucent with clear cell boundaries. As shown in Figure 1A, ESC clonal colonies in the GE co-culture group were irregular in shape with rough boundaries, which were faintly visible. U118 cells in the TMZ group were in a fair condition and there were significantly more dead cells than in the control group. Similarly, U118 cells co-cultured with ESC had poor translucency, and the number of dead cells significantly increased.

To investigate whether the role of the ESC microenvironment on U118 cells was down-regulated by the PI3K pathway, we examined PI3K pathway-related genes and found that there was a decrease in the expression of PI3K, AKT, and mTOR in U118 cells, while the expression of the PI3K inhibitor, PTEN, increased after co-culture with ESC (as shown in Figure 1B,C). When the PI3K pathway agonist (PTEN inhibitor), SF1670, was added to the GE co-culture group, the expression of cell proliferation-promoting proteins $\mathrm{PI} 3 \mathrm{~K}, \mathrm{AKT}$, and mTOR increased.

\section{ESC microenvironment inbibited the proliferative ability of $U 118$ cells}

As displayed in Figure 2A, the results of CCK8 proliferation assays showed the proliferation ability and cell growth curve of U118 cells in each group. In the incubation period, when growth was slow, there were no significant differences in the growth of the four groups of U118 cells. The growth curve of U118 cells was markedly smaller in the GE coculture group than in the control group, but there was no significant difference between the GT chemotherapypositive control group and the co-culture group.

Clone formation assays showed that the clone formation rate of the GE group $(10 \% \pm 0.26 \%)$ was lower than that of the control group $(29 \% \pm 0.2 \%)$, and the difference was statistically significant $(\mathrm{P}<0.01)$. This indicates that ESC microenvironment can inhibit the clonogenicity of U118 cells (as shown in Figure 2B-2C).

To observe the role of the ESC microenvironment on the cell cycle of U118 cells, we used a flow cytometer to measure the cell cycle distribution of each group of U118 cells. The results showed a significant reduction in the proportion of U118 cells in the GE co-culture group that exited the G1 phase and entered the replicationproliferation phase $(\mathrm{S}+\mathrm{G} 2$ phase). In particular, the number of cells entering the $S$ phase $(14.91 \% \pm 10.84 \%)$ was significantly lower than that in the control group $(25.50 \% \pm 19.92 \%, \mathrm{P}<0.05$; as shown in Figure $2 D)$. To understand the effectors that influence the cell cycle, we examined a number of factors related to the cell cycle. QRT-PCR results showed that the expression of cell cycle proteins cyclin B1 and cyclin D1 decreased in U118 cells co-cultured with ESC, as well as a notable increase in the expression of p27, p2 1 and GSK-3 $\beta$ negative cell cycle regulators. The changes in these genes were consistent with cell cycle measurement results (as shown in Figure 2E). Western blotting analysis confirmed that the expression of cyclin D1 and cyclin B1 cell cycle proteins decreased in U118 cells co-cultured with ESC, and that the expression of negative cell cycle regulators p27, p21, and GSK-3 $\beta$ increased substantially (as shown in Figure $2 F$ ). There was no change in the cell cycle-related protein expression of U118 cells in the GT chemotherapy group.

\section{ESC microenvironment promoted apoptosis of U118 cells}

ROS promote apoptosis through the p53/Bax pathway, ultimately leading to cell death. A flow cytometer was used to detect the ROS expression level in each group (as shown in Figure $3 A, B)$. It could be seen that the level of ROS was significantly higher in the GE co-culture group than that in the control group $(\mathrm{P}<0.05)$. The status of apoptosis and necrosis of U118 cells in each group was determined. The apoptosis rates of U118 cells in the GB control group, GE co-culture group, and GT chemotherapy group were $12.64 \% \pm 0.285 \%, 39.6 \% \pm 5.015 \%$, and $48.1 \% \pm 4.9529 \%$, 

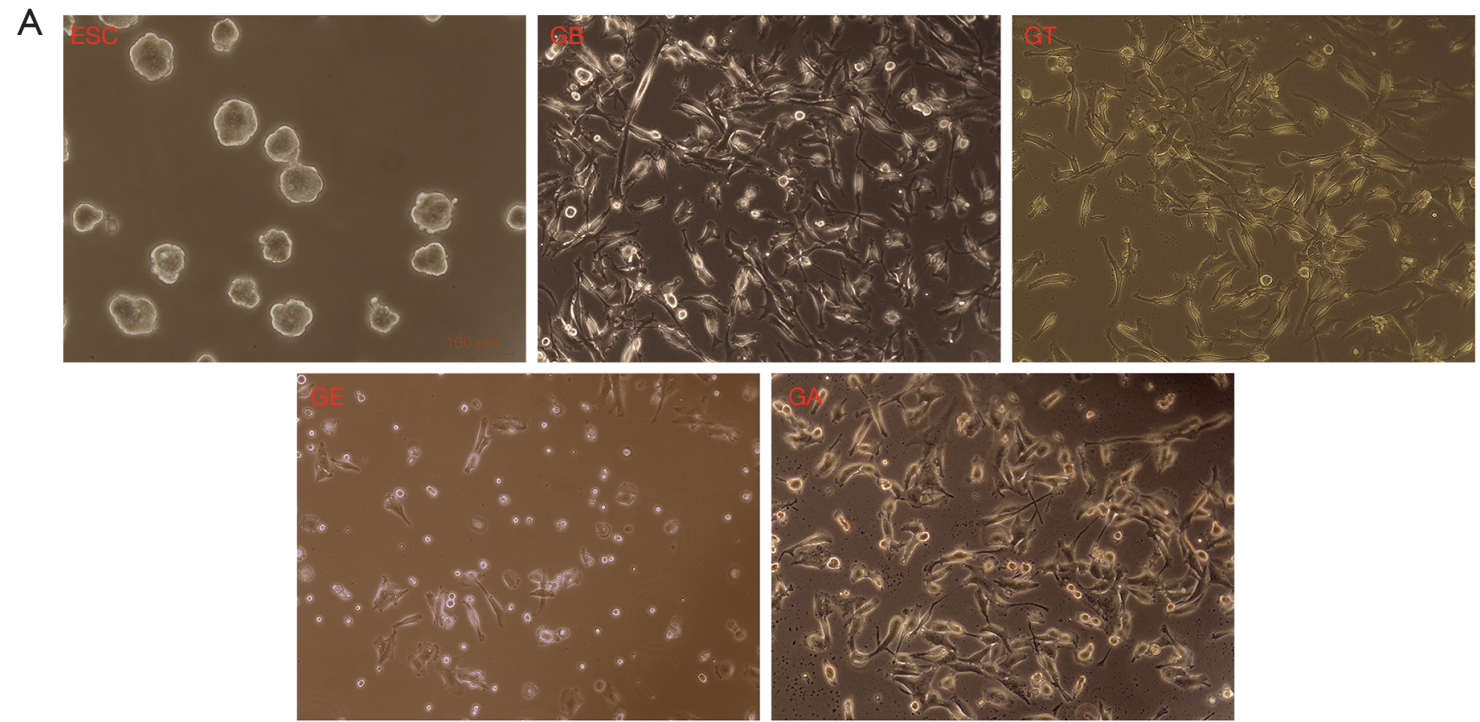

B
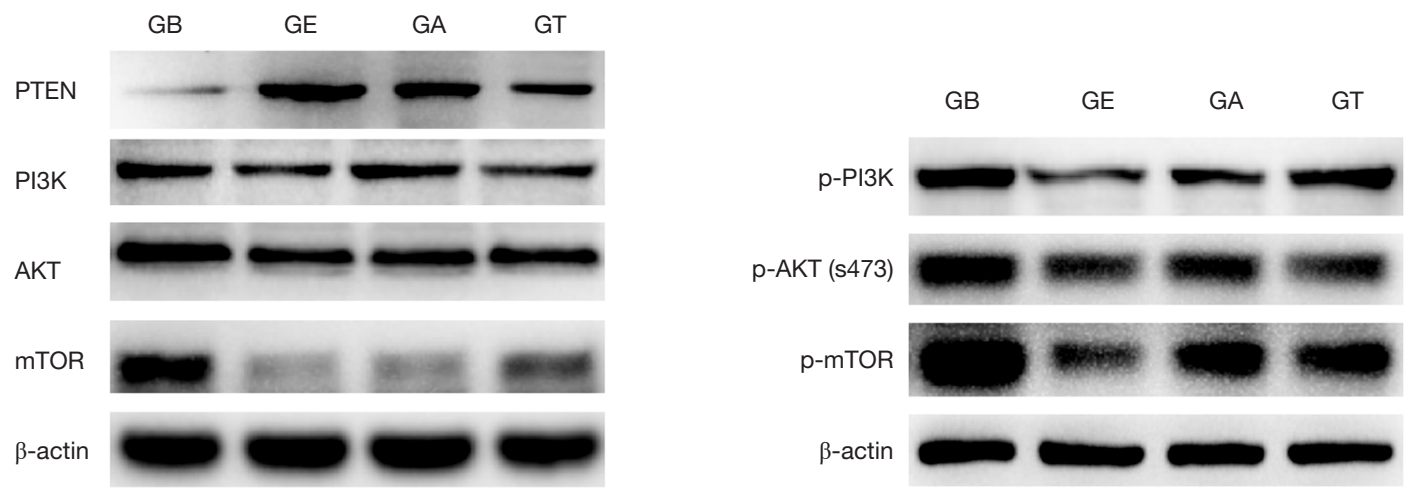

C

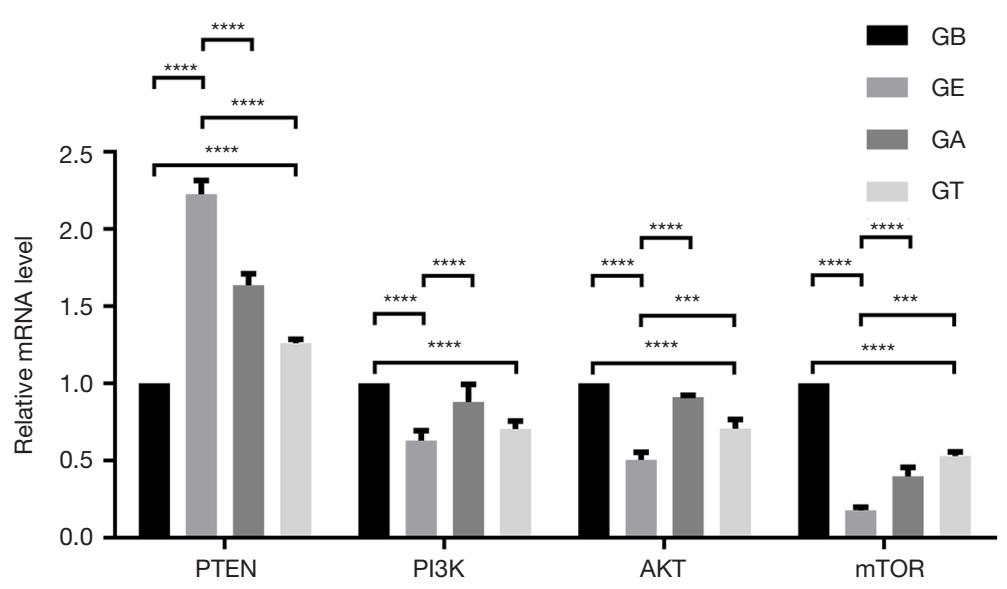

Figure 1 The embryonic stem cell (ESC) microenvironment increases the proliferative ability of U118 cells by down-regulating the PI3K pathway. (A) The condition of each group of cells observed by inverted microscope with white light shooting, 200 times lens shooting; (B) PI3K/AKT signaling pathway protein expression level in GB, GE, GA, GT; (C) the mRNA expression level of PI3K/AKT signaling pathway in GB, GE, GA, GT. ES, embryonic stem cells; GB, U118 cells; GE, U118 Co-culture ESC; GA, U118 co-culture ESC plus PTEN inhibitor; GT, U118 plus temozolomide. ${ }^{* * *} \mathrm{P}<0.001,{ }^{* * *} \mathrm{P}<0.0001$. 

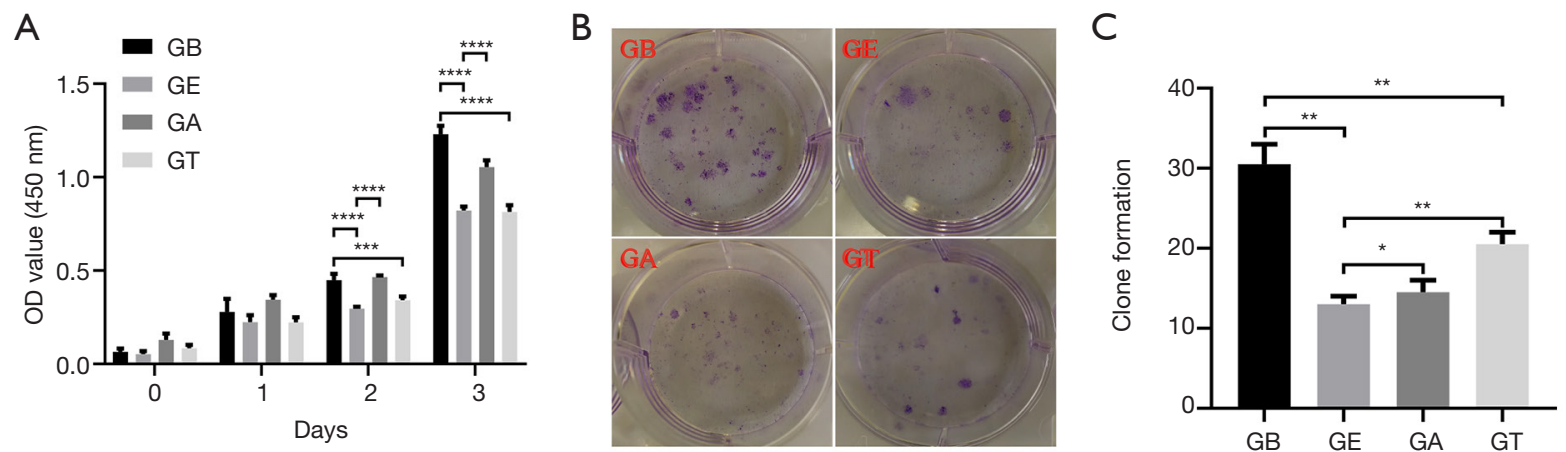

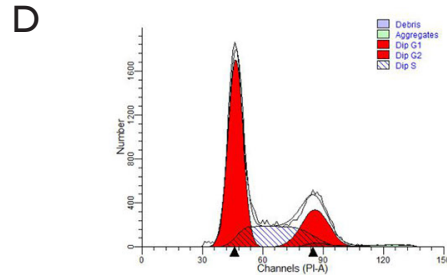

$\mathrm{GB}$

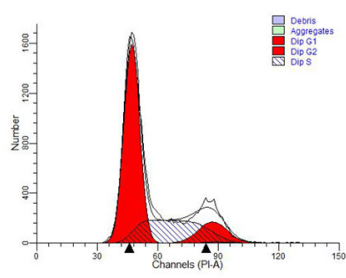

GA

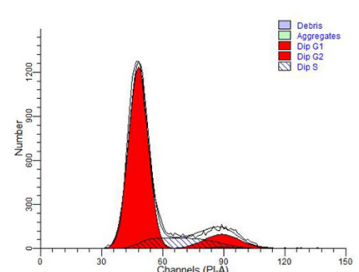

GE

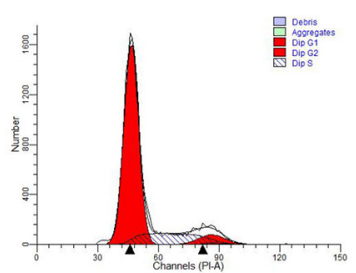

GT

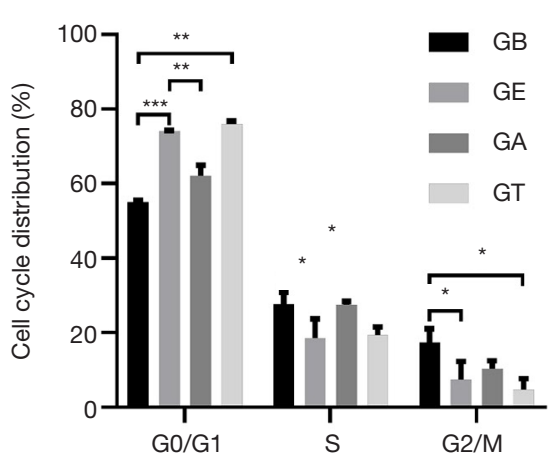

$\mathrm{E}$

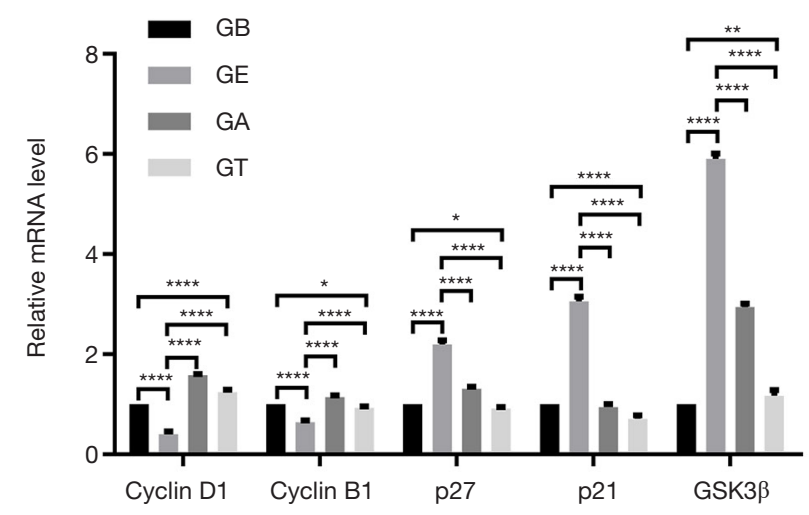

$\mathrm{F}$

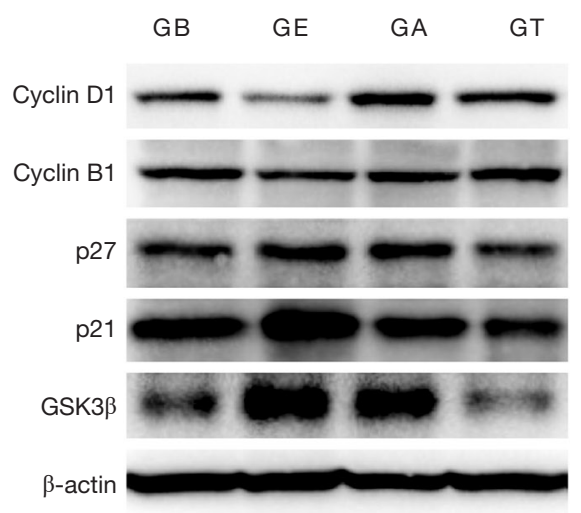

Figure 2 The embryonic stem cell (ESC) microenvironment inhibits the proliferative ability of U118 cells. (A) CCK8 experiment detects the proliferation ability of cells between different groups. (B,C) ES microenvironment can inhibit the clonogenicity of U118 cells with crystal violet stain. (D) Cell flow cytometry detects cell cycle changes between different groups. (E) Changes in the expression levels of these cycle-related genes in different groups of U118 cells. (F) Changes in the expression levels of these cycle-related proteins in different groups of U118 cells. ${ }^{*} \mathrm{P}<0.05,{ }^{* *} \mathrm{P}<0.01,{ }^{* * *} \mathrm{P}<0.001,{ }^{* * * *} \mathrm{P}<0.0001$. 
A

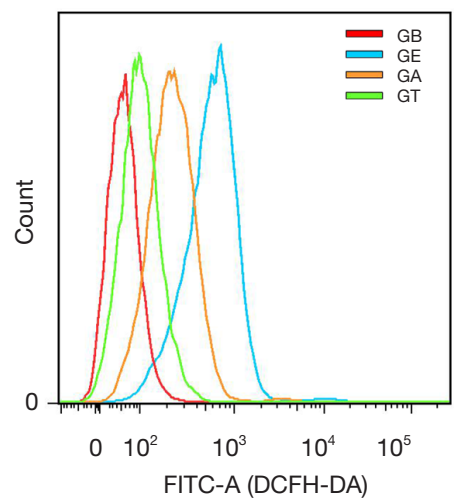

B

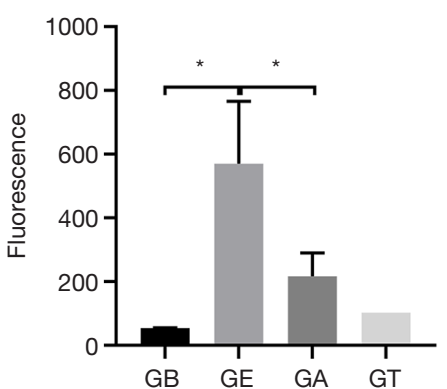

D

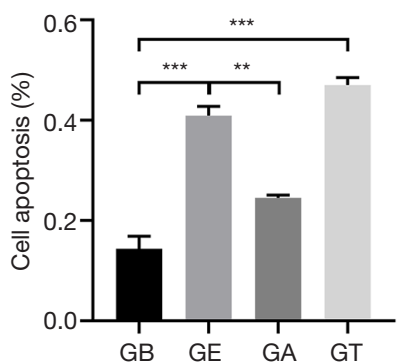

C
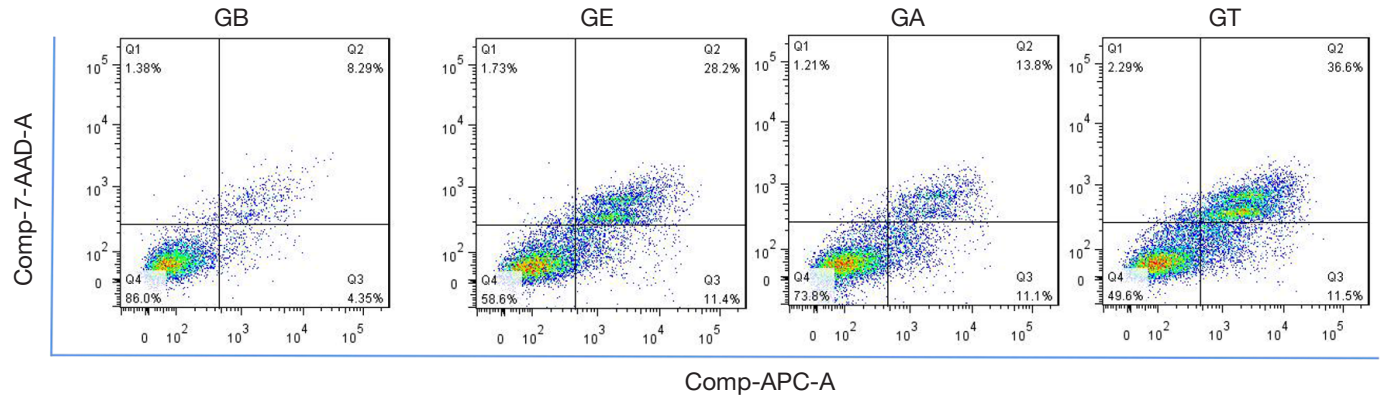

E
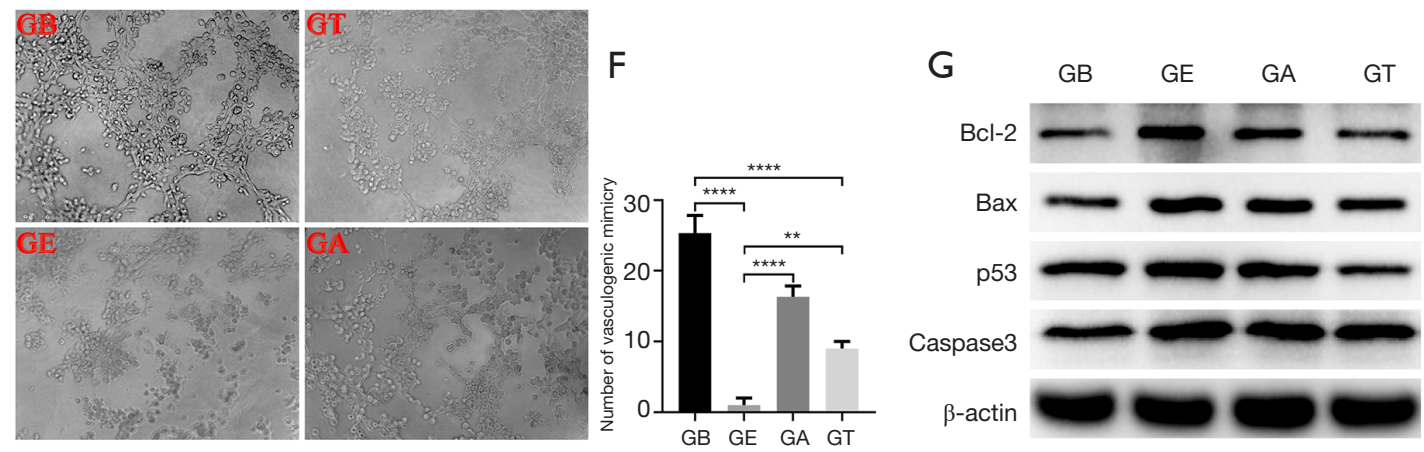

$\mathrm{H}$

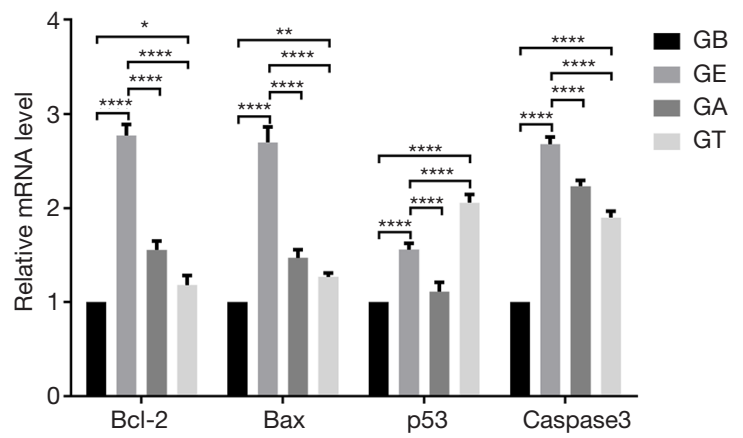

I

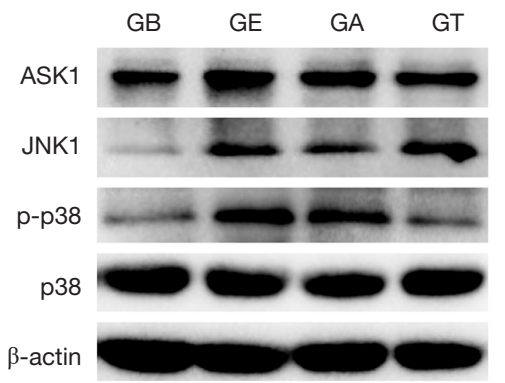

Figure 3 The embryonic stem cell (ESC) microenvironment promotes apoptosis of U118 cells. (A,B) Flow cytometer was used to detect the expression level of ROS in each group. (C,D) The apoptosis rate of U118 cells in each group. (E,F) ESC microenvironment blocked U118 cells vasculogenic mimicry formation inverted microscope with white light shooting, 200 times lens shooting. (G) The pro-apoptosis protein Bcl-2, p53, Bax and Caspase-3 expression levels in each group. (H) The pro-apoptosis genes Bcl-2, p53, Bax and Caspase-3 expression levels in each group. (I) The expression of ROS-related proteins ASK1, JNK1, p38, and p-p38 in each group. ${ }^{*} \mathrm{P}<0.05,{ }^{* *} \mathrm{P}<0.01,{ }^{* * *} \mathrm{P}<0.001,{ }^{* * *} \mathrm{P}<0.0001$. 
respectively. The apoptosis rate of $\mathrm{U} 118$ co-cultured with ESC increased considerably, while the apoptosis rate of U118 cells was highest in the GT chemotherapy group, indicating that the ESC microenvironment can promote the apoptosis of U118 cells (as shown in Figure 3C,D). The ESC microenvironment also blocked U118 cells vasculogenic mimicry formation (shown in Figure 3E,F).

We also measured the changes in genes associated with apoptosis. In the GB group, the expression of the pro-apoptosis genes Bcl-2, p53, Bax, and Caspase-3 were significantly higher compared to the control group, although there was no significant difference compared to the chemotherapy group. This indicated that the GE coculture group promoted tumor cell apoptosis consistent with the effects of chemotherapy agents. Western blotting confirmed that the expression of pro-apoptosis genes Bcl2, p53, Bax, and Caspase- 3 in U118 cells in the GE coculture group was markedly higher than the control group (as shown in Figure 3G,H). Relevant studies have found that after ROS increases in cells, it can regulate cell apoptosis by activating the ASK1/JNK1 and p38MAPK pathways (15-17). We detected the expression levels of ASK1, JNK1, p38 and p-p38 by western, and found that the expression levels of ASK1, JNK1 and phosphorylated p38 in GE group were significantly up-regulated compared with GB group (as shown in Figure 3I).

\section{Discussion}

Gliomas are tumors derived from neuroepithelial cells and account for $40-50 \%$ of all intracranial tumors. They have high rates of incidence, recurrence, and mortality, as well as a low cure rate (18). At present, TMZ is used in the clinical treatment of glioma (19). Studies have reported that TMZ can inhibit the proliferation of glioma cells, effectively promoting apoptosis and blocking the cell cycle. In the present study, the CCK8 proliferation test of U118 cell clones in the TMZ chemotherapy group showed a significant decrease in cell proliferation, and the proportion of apoptosis increased (confirmed by ROS and apoptosis tests). Recurrent GBM tumors are usually resistant to TMZ, and although patients are relatively well tolerated to TMZ, there are still dose-dependent adverse reactions, such as severe bone marrow suppression, which limits the TMZ treatment dose (20).

The embryonic microenvironment can effectively regulate the biological behavior of tumor cells and completely reverse tumor cells into benign cells. The ability of the embryonic microenvironment to reverse tumor cells gradually diminishes as the embryo develops and differentiates. Gerschenson et al. (21) injected melanoma cells into E10 mouse embryos (12-14), E14 mouse embryos, and the dorsal skin of newborn mice. The tumor formation rates were $0(0 / 5), 73 \%(32 / 44)$, and $80 \%(16 / 20)$, respectively. Cucina et al. (22) found that extract from late zebrafish blastocysts could inhibit growth and proliferation and promote the apoptosis of human rectal cancer cells, while extract from embryos at the gastrula stage had no such effect. Postovit et al. (23) found that treatment with adult bone marrow stem cell, amniotic fluid stem cell, and trophoblast cell microenvironments did not have tumor-reversing effects. These results indicate that only the embryonic microenvironment has tumor-reversing effects. Previous studies have also found that ESC can recapitulate the microenvironment of embryos. We attempted to use ESC on a mouse leukemia model and found that ESC could significantly improve myelography of mice and prolong their survival. In addition, we observed that ESC could reverse the degree of malignancy of uveal and cutaneous melanomas in vitro. Therefore, to simulate this early embryonic microenvironment more efficiently, ESCs were selected as the effector cells in our study instead of other types stem cells (like bone marrow mesenchymal stem cells) to simulate the embryonic microenvironment. Our study showed that tumor growth significantly reduced, apoptotic cells increased, and clonogenicity decreased after co-culture with ESC.

Primary GBM has three core signaling pathways: receptor tyrosine kinase (RTK)/PI3K/mitogen activated protein kinase (MAPK), retinoblastoma, and tumor protein $\mathrm{p} 53$. The RTK/PI3K/MAPK pathway contains mutations in $90 \%$ of cases of GBM (24). MAPK and PI3K promote the growth of many cancers, including migration, proliferation, and survival $(25,26)$. The tumor suppressor, PTEN, affects cell functions such as cell growth, proliferation, survival, and migration, and is a core negative regulator of the PI3K/AKT signaling pathway. The loss of PTEN also fails to block RTK inhibitors to turn off PI3K signaling, however may reduce the likelihood that a single drug will cause cancer to respond to these treatments $(27,28)$. The present study revealed that in the ESC and U118 GE cocultured group of glioblastoma cells, PTEN expression was significantly increased, PI3K and AKT expression was markedly reduced. ESC can promote PTEN expression and inhibit PI3K signaling pathway activation, thereby reversing the degree of malignancy of U118 cells. At the same time, PTEN expression was decreased in the GA group with the addition of a PI3K agonist, but was increased in the 
control group. The expression of PI3K, AKT, and mTOR was higher in the GA group than that in the GE co-culture group, indicating that the ESC inhibited the PI3K/AKT signaling pathway to reduce the degree of malignancy of U118 cells.

ROS promote apoptosis through the p53/Bax pathway (29) and leads to cell death. The p53 protein inhibits cell cycle progression and induces apoptosis, and AKT activation inhibits p53-mediated apoptosis (30). When AKT is inactivated, cell mitochondrial membrane potential is lost, activating Caspase-9 and Caspase-3, which leads to apoptosis (31). TMZ can inhibit the normal function of the mitochondrial respiratory chain by interfering with oxidative phosphorylation, leading to ATP deficiency, ROS formation, and oxidative stress. In the present study, both the ESC co-culture group and the TMZ induction group were found to promote the apoptosis of U118 cells. The ESC microenvironment could promote the increase in ROS in U118 cells, leading to oxidative damage of the cells, and an increase in pro-apoptosis proteins $\mathrm{p} 53$, Bax, and Caspase-3, resulting in increased apoptosis of $\mathrm{U} 118$ cells. In addition, when the PI3K agonist was added to the co-culture system, the expression of ROS in tumor cells significantly reduced, indicating that the ESC microenvironment reduces oxidative damage in U118 cells by down-regulating the PI3K pathway.

GSK-3 $\beta$ can inhibit tumor transformation and development, and is considered to be a tumor suppressor (32). Hepatocellular carcinoma cells are resistant to etoposide and camptothecin after treatment with the GSK- $3 \beta$ inhibitor, lithium. However, the expression of GSK-3 $\beta$ activated by LY294002 and exogenous Ser9 GSK-3 $\beta$ can increase these drugs to induce hepatocellular carcinoma cell apoptosis (33). Other reports suggest that GSK-3 $\beta$ activation can promote sensitivity of human breast cancer cells to paclitaxel, 5-fluorouracil, cisplatin, and prodigiosin (34). In addition, studies have shown that inhibition of GSK$3 \beta$ phosphorylation can significantly reduce tumor aggressiveness (35). The cell cycle proteins, cyclin D1 and cyclin B1, play important roles in cell cycle tumorigenesis and regulation. Cyclin B1 is a G2 phase cyclin, which increases the acceleration of G2/M phase transition and causes abnormal cell proliferation. Cyclin D1 is the most important regulatory factor at the G1/S checkpoint, and acts in the G1 phase to promote cell entry into the $S$ phase in conjunction with many associated proteins, and promotes cell division and proliferation. The present study found that the ESC microenvironment could promote the expression of cell cycle-associated proteins that inhibit proliferation by significantly increasing the expression of GSK-3 $\beta, \mathrm{p} 21$, and $\mathrm{p} 27$, while reducing the expression of cell cycle proteins associated with tumor proliferation, including cyclin D1 and cyclin B1. After addition of the PI3K agonist, the expression of cell cycle-associated proteins that inhibit the proliferation of GSK-3 $\beta$, p21, and p27 in tumor cells substantially decreased, while the expression of cell cycle proteins associated with tumor proliferation, such as cyclin D1 and cyclin B1, markedly increased. This demonstrates that the ESC microenvironment can inhibit the cell cycle of U118 cells by down-regulating PI3K.

The homing of stem cells has to intracranial lesions makes them more suitable for residual and primary GBM (36). Using tumor-specific therapeutic proteins and advanced imaging agents, it has been demonstrated that both human and mouse stem cells can home to GBM and play a therapeutic role $(37,38)$. A recent study showed that the growth and resection of malignant GBM cells in vivo could be observed in real time through the use of fluorescent and bioluminescent protein markers, thus mimicking the clinical status of GBM resection. The main barriers preventing many drugs from reaching brain tumor cells are the blood-brain barrier (BBB) (39) and tumor vascular dysfunction (40). One solution to intracranial tumor drug administration is to provide new tumor-specific drugs in situ. This study shows that the ESC microenvironment can significantly promote tumor cell apoptosis and inhibit tumor cell proliferation. The ESC co-culture group and the TMZ chemotherapy group promoted ROS expression, and $\mathrm{PI} 3 \mathrm{~K} / \mathrm{AKT} / \mathrm{mTOR}$ promoted decreased expression of tumor proliferation proteins and inhibited the increased expression of tumor proliferating proteins p21, p27, and GSK-3 $\beta$.

\section{Conclusions}

The present study found that the ESC microenvironment could inhibit the PI3K/AKT pathway to promote apoptosis and inhibit U118 cells proliferation. These findings provide a new approach for the development of ESC transplantation in cancer treatment by reversing the microenvironment of cancer survival rather than directly killing tumor cells.

\section{Acknowledgments}

The authors thank Professor Andy Peng Xiang, from the Center for Stem Cell Biology and Tissue Engineering, the 
Key Laboratory for Stem Cells and Tissue Engineering, Ministry of Education, Sun Yat-Sen University for providing mouse ESC, This work was supported by This work was supported by The National Key R\&D program of China (2018YFC1106000) and Miao Miao cultivation plan start-up project of Shenzhen Hospital, Southern Medical University, 2018MM14; Sanming Project of Medicine in ShenZhen SZSM201812047.

\section{Footnote}

Reporting Checklist: The authors have completed the MDAR reporting checklist. Available at http://dx.doi. org/10.21037/tcr-20-3408

Data Sharing Statement: Available at http://dx.doi. org/10.21037/tcr-20-3408

Conflicts of Interest: All authors have completed the ICMJE uniform disclosure form (available at http://dx.doi. org/10.21037/tcr-20-3408). The authors have no conflicts of interest to declare.

Ethical Statement: The authors are accountable for all aspects of the work in ensuring that questions related to the accuracy or integrity of any part of the work are appropriately investigated and resolved.

Open Access Statement: This is an Open Access article distributed in accordance with the Creative Commons Attribution-NonCommercial-NoDerivs 4.0 International License (CC BY-NC-ND 4.0), which permits the noncommercial replication and distribution of the article with the strict proviso that no changes or edits are made and the original work is properly cited (including links to both the formal publication through the relevant DOI and the license). See: https://creativecommons.org/licenses/by-nc-nd/4.0/.

\section{References}

1. Thakkar JP, Dolecek TA, Horbinski C, et al. Epidemiologic and molecular prognostic review of glioblastoma. Cancer Epidemiol Biomarkers Prev 2014;23:1985-96.

2. Nabors LB, Portnow J, Ahluwalia M, et al. Central Nervous System Cancers, Version 3.2020, NCCN Clinical Practice Guidelines in Oncology. J Natl Compr Canc Netw. 2020;18:1537-70.

3. DeGregori J. Connecting Cancer to Its Causes Requires
Incorporation of Effects on Tissue Microenvironments. Cancer Res 2017;77:6065-8.

4. Bissell MJ, Hines WC. Why don't we get more cancer? A proposed role of the microenvironment in restraining cancer progression. Nat Med 2011;17:320-9.

5. Lee LM, Seftor EA, Bonde G, et al. The fate of human malignant melanoma cells transplanted into zebrafish embryos: assessment of migration and cell division in the absence of tumor formation. Dev Dyn 2005;233:1560-70.

6. Kulesa PM, Kasemeier-Kulesa JC, Teddy JM, et al. Reprogramming metastatic melanoma cells to assume a neural crest cell-like phenotype in an embryonic microenvironment. Proc Natl Acad Sci U S A 2006;103:3752-7.

7. Astigiano S, Damonte P, Fossati S, et al. Fate of embryonal carcinoma cells injected into postimplantation mouse embryos. Differentiation 2005;73:484-90.

8. Haldi M, Ton C, Seng WL, et al. Human melanoma cells transplanted into zebrafish proliferate, migrate, produce melanin, form masses and stimulate angiogenesis in zebrafish. Angiogenesis 2006;9:139-51.

9. Shi Y, Du L, Lin L, et al. Tumour-associated mesenchymal stem/stromal cells: emerging therapeutic targets. Nat Rev Drug Discov 2017;16:35-52.

10. Giuffrida D, Rogers IM, Nagy A, et al. Human embryonic stem cells secrete soluble factors that inhibit cancer cell growth. Cell Prolif 2009;42:788-98.

11. Postovit LM, Seftor EA, Seftor RE, et al. A threedimensional model to study the epigenetic effects induced by the microenvironment of human embryonic stem cells. Stem Cells 2006;24:501-5.

12. Zhou C, Huang Z, Li P, et al. Safety and efficacy of embryonic stem cell microenvironment in a leukemia mouse model. Stem Cells Dev 2014;23:1741-54.

13. Liu J, Huang Z, Yang L, et al. Embryonic Stem Cells Modulate the Cancer-Permissive Microenvironment of Human Uveal Melanoma. Theranostics 2019;9:4764-78.

14. Wang C, Wang X, Liu J, et al. Embryonic stem cell microenvironment suppresses the malignancy of cutaneous melanoma cells by down-regulating PI3K/AKT pathway. Cancer Med 2019;8:4265-77.

15. Sablina AA, Budanov AV, Ilyinskaya GV, et al. The antioxidant function of the p53 tumor suppressor. Nat Med 2005;11:1306-13.

16. Fang XY, Zhang $\mathrm{H}$, Zhao L, et al. A new xanthatin analogue 1beta-hydroxyl-5alpha-chloro-8-epi-xanthatin induces apoptosis through ROS-mediated ERK/p38 MAPK activation and JAK2/STAT3 inhibition in human 
hepatocellular carcinoma. Biochimie 2018;152:43-52.

17. Park SG, Kim SH, Kim KY, et al. Toyocamycin induces apoptosis via the crosstalk between reactive oxygen species and p38/ERK MAPKs signaling pathway in human prostate cancer PC-3 cells. Pharmacol Rep 2017;69:90-6.

18. Sathornsumetee S, Rich JN, Reardon DA. Diagnosis and treatment of high-grade astrocytoma. Neurol Clin. 2007;25:1111-39, x.

19. Li G, Zhang H, Liu Y, et al. Effect of temozolomide on livin and caspase-3 in U251 glioma stem cells. Exp Ther Med 2015;9:744-50.

20. Jiang G, Wei ZP, Pei DS, et al. A novel approach to overcome temozolomide resistance in glioma and melanoma: Inactivation of MGMT by gene therapy. Biochem Biophys Res Commun 2011;406:311-4.

21. Gerschenson M, Graves K, Carson SD, et al. Regulation of melanoma by the embryonic skin. Proc Natl Acad Sci U S A 1986;83:7307-10.

22. Cucina A, Biava PM, D'Anselmi F, et al. Zebrafish embryo proteins induce apoptosis in human colon cancer cells (Caco2). Apoptosis 2006;11:1617-28.

23. Postovit LM, Margaryan NV, Seftor EA, et al. Human embryonic stem cell microenvironment suppresses the tumorigenic phenotype of aggressive cancer cells. Proc Natl Acad Sci U S A 2008;105:4329-34.

24. Brennan CW, Verhaak RG, McKenna A, et al. The somatic genomic landscape of glioblastoma. Cell 2013;155:462-77.

25. Hanahan D, Weinberg RA. Hallmarks of cancer: the next generation. Cell 2011;144:646-74.

26. Thorpe LM, Yuzugullu H, Zhao JJ. PI3K in cancer: divergent roles of isoforms, modes of activation and therapeutic targeting. Nat Rev Cancer 2015;15:7-24.

27. Mellinghoff IK, Wang MY, Vivanco I, et al. Molecular determinants of the response of glioblastomas to EGFR kinase inhibitors. N Engl J Med 2005;353:2012-24.

28. Berns K, Horlings HM, Hennessy BT, et al. A functional genetic approach identifies the PI3K pathway as a major determinant of trastuzumab resistance in breast cancer. Cancer Cell 2007;12:395-402.

29. Davis RJ. Signal transduction by the JNK group of MAP

Cite this article as: He X, Liu J, Wang X, Liu T, Yang L, Li C, Wang C, Liu Y, Sang X, Wang Z, Lu X. The embryonic stem cell microenvironment inhibits mouse glioma cell proliferation by regulating the PI3K/AKT pathway. Transl Cancer Res 2021;10(1):487-498. doi: 10.21037/tcr-20-3408 kinases. Cell 2000;103:239-52.

30. Oren M. Decision making by p53: life, death and cancer. Cell Death Differ 2003;10:431-42.

31. Nikoletopoulou V, Markaki M, Palikaras K, et al. Crosstalk between apoptosis, necrosis and autophagy. Biochim Biophys Acta 2013;1833:3448-59.

32. Medina M, Wandosell F. Deconstructing GSK-3: The Fine Regulation of Its Activity. Int J Alzheimers Dis 2011;2011:479249.

33. Rocha CR, Garcia CC, Vieira DB, et al. Glutathione depletion sensitizes cisplatin- and temozolomideresistant glioma cells in vitro and in vivo. Cell Death Dis 2014;5:e1505.

34. Luo J. Glycogen synthase kinase 3 beta (GSK3beta) in tumorigenesis and cancer chemotherapy. Cancer Lett 2009;273:194-200.

35. Pyko IV, Nakada M, Sabit H, et al. Glycogen synthase kinase 3 beta inhibition sensitizes human glioblastoma cells to temozolomide by affecting O6-methylguanine DNA methyltransferase promoter methylation via c-Myc signaling. Carcinogenesis 2013;34:2206-17.

36. Corsten MF, Shah K. Therapeutic stem-cells for cancer treatment: hopes and hurdles in tactical warfare. Lancet Oncol 2008;9:376-84.

37. Balyasnikova IV, Ferguson SD, Han Y, et al. Therapeutic effect of neural stem cells expressing TRAIL and bortezomib in mice with glioma xenografts. Cancer Lett 2011;310:148-59.

38. Menon LG, Kelly K, Yang HW, et al. Human bone marrow-derived mesenchymal stromal cells expressing S-TRAIL as a cellular delivery vehicle for human glioma therapy. Stem Cells 2009;27:2320-30.

39. Jain RK, di Tomaso E, Duda DG, et al. Angiogenesis in brain tumours. Nat Rev Neurosci 2007;8:610-22.

40. Takahashi K, Yamanaka S. Induction of pluripotent stem cells from mouse embryonic and adult fibroblast cultures by defined factors. Cell 2006;126:663-76.

(English Language Editor: A. Kassem) 